\title{
A further study of interchromosomal epistatic interaction in male mating activity of Drosophila melanogaster
}

\author{
KAZUHIKO KOSUDA \\ Biological Laboratory, Josai University, Sakado, Saitama, Japan 350-02
}

\begin{abstract}
The interchromosomal epistatic interaction between second and third chromosomes was investigated for male mating activity of Drosophila melanogaster. The observed mating activity of double chromosome homozygotes is $0.526 \pm 0.044$, whereas the expected one under the multiplicative model is calculated to be $0.511 \pm 0.038$. These two male mating activities are in excellent agreement, indicating no interchromosomal epistasis. The coefficient of interaction, $i$, is quantified as $-0.174 \pm 0.168$. The magnitude of the $k$ statistic, another index of epistasis, is $0.0001 \pm 0.0267$. Neither index of epistasis differs statistically from zero. The result also shows that there is little or no epistatic interaction between two major chromosomes. However, one interchromosomal synthetic sterility was found in 26 chromosome lines. These results are combined with those of Kosuda (1985) and the generality of epistatic interaction is discussed.
\end{abstract}

Keywords: chromosome, Drosophila melanogaster, interaction, mating activity, synthetic sterility.

\section{Introduction}

If positive interaction in fitness predominates in natural populations, a greater number of polymorphisms can be maintained by balancing selection. To what extent do fitness differentials between multi-locus genotypes depend upon epistatic interaction is therefore one of the important questions in population genetics. Experimental evaluation of epistatic interaction has been carried out for egg-to-adult viability in some Drosophila species (Spassky et al., 1965; Temin et al., 1969; Kosuda, 1971). Fitness components other than preadult viability have been neglected in this kind of approach to the problem. Further approaches should be made for other components of fitness, as Prout (1965) showed that the estimation of net fitness is best done by splitting it into various components. Furthermore, evidence has accumulated that the male reproductive component of fitness has a much greater effect on total fitness than egg-to-adult viability and female fertility in estimating selective differences (Anderson, 1969; Petit et al., 1980; Brittnacher, 1981; Kosuda, 1983; Sharp, 1984).

In a previous study, Kosuda (1985) reported that there is almost no interchromosomal interaction between the second and third chromosome of $D$. melanogaster for male mating activity. This conclusion, however, has received some criticism, as the number of experimental chromosome lines was limited and the mating activity was calculated from a relatively small number of observations in comparison with viability estimates. A further attempt to evaluate the intensity of epistatic interaction between genes on different chromosomes for the male mating activity of $D$. melanogaster has been carried out and the results will be discussed together with those from the previous study.

\section{Materials and methods}

Experimental materials and methods are the same as in the previous study (Kosuda, 1985). Wild flies of Drosophila melanogaster from a natural population in Katsunuma. Yamanashi, Japan were used. Pairs of second and third chromosomes were extracted from male flies in the natural population by mating with $C y /$ $P m ; U b x / S b$ females, and were made homozygous by means of the routine marked inversion method (Fig. 1). Flies expressing both $C y$ and $U b x$ phenotype in the $\mathrm{G}_{4}$ generation are doubly heterozygous for second and third chromosomes from the natural population. $U b x$ and $C y$ flies are singly homozygous for either second or third chromosomes respectively. Flies with a wild phenotype are double homozygotes. Many chromo- 


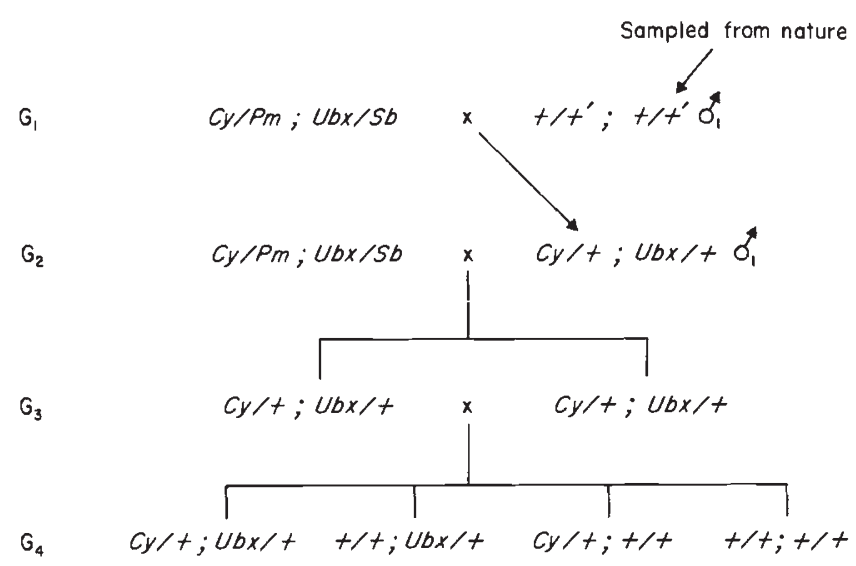

Fig. 1 Mating scheme to obtain the double chromosome homozygotes and each of single chromosome homozygotes for second and third chromosomes, and the double heterozygotes.

somes extracted from the natural population were homozygous lethals or semilethals. These chromosomes were excluded from the experiment. Chromosomes carrying alleles for sterility and low mating activity were also omitted.

Non-competitive male mating activity of various homozygotes was quantified for the 26 chromosome lines thus established as follows. Male flies were singly placed into a $3 \times 8 \mathrm{~cm}$ mating vial with food and containing 10 virgin females from a standard laboratory strain, 2SG, aged 3-4 days. They were kept at $25^{\circ} \mathrm{C}$ under continuous light. Male flies were discarded after $24 \mathrm{~h}$, and females were dissected under the microscope to examine the presence of sperm in ventral receptacles or spermathecae. Between five and 10 replicates were made for each homozygous chromosome line. Male mating activity was defined as the proportion of inseminated female flies.

Mating activity of various heterozygous males was also measured in the same way, using male progenies made by crosses between $C y$; $U b x$ females and males derived from the homozygous chromosome lines. Thus the wild type flies in these controls have two different second and third chromosomes and consequently the inbreeding coefficient $(F)$ is 0 .

To calculate the intensity of interchromosomal interaction in male mating activity, two indices, $i$ and $k$ statistics, were employed. The coefficient of interaction, $i$, was adopted by Spassky et al. (1965), Temin et al. (1968) and Kosuda $(1971,1985)$ and the $k$ statistic was devised by Seager \& Ayala (1982) and employed by Seager et al. (1982) and Kosuda (1985).

$1-i$ is defined by the observed mating activity divided by the expected mating activity, that is, $1-i=w X Y z / W x y Z$, where $W, X, Y, Z$ are the mating activities of heterozygous males $(F=0)$ and $w, x, y, z$ are the mating activities of homozygotes $(F=1)$ of $C y$; $U b x, U b x, C y$, and wild type, respectively. If there is no interchromosomal epistatic interaction, $i$ should be 0 . When $i>0$, positive epistatic interaction (reinforcing or synergistic interaction) exists, that is, the mating activity of double chromosome homozygotes is lower than that expected under the multiplicative model. A higher-than-expected mating activity for double chromosome homozygotes gives $i<0$. As the range of $i$ is $-1 \leqslant i \leqslant 1$, the $i$ statistic has defective properties in that it is not symmetrical around 0 and responds too much to random fluctuations in the measurement of mating activity. In comparison with the viability estimation, mating activity is measured for a relatively limited number of observations, and the experimental chromosome lines are limited. Therefore, the $i$ statistic is not an appropriate estimate in variable traits such as male mating activity. To overcome the difficulty, Seager \& Ayala (1982) devised the $k$ statistic, a correlation between heterozygosity and homozygosity for two chromosomes. If the effect of homozygosity for one chromosome does not depend upon the homozygosity in the other, there will be no correlation and $k$ should be zero. If two chromosomes interact, i.e. if there is an association of fitness effect for homozygosity of the two chromosomes, then a positive correlation coefficient will be obtained. The magnitude of the correlation, $r$, is a measure of interchromosomal epistasis. The $k$ statistic is symmetric about zero and its range is $-1 \leqslant k \leqslant 1$. The sign of $k$ is the same as for the $i$ statistic. $k$ is calculated by the following formula:

$$
\begin{aligned}
k=-r & =d-(b+d)(c+d) /\left\{\left[b+d-(b+d)^{2}\right]\right. \\
& \left.\times\left[c+d-(c+d)^{2}\right]\right\},
\end{aligned}
$$

where $a, b, c$ and $d$ are the standardized relative mating activity of $C y ; U b x, U b x, C y$ and wild type, respectively, so that they add up to 1.0 .

\section{Results}

More than 14,000 female flies in total were dissected to examine the male mating activities in chromosomally homozygous and heterozygous states. These mating activities are presented in Table 1 . The relative male mating activities of double homozygotes and each of single homozygotes for second and third chromosomes to the double heterozygotes are calculated to be $0.552 \pm 0.059, \quad 0.764 \pm 0.035$ and $0.647 \pm 0.052$, respectively. The effect of marker chromosomes is thus taken into consideration in the estimation of the relative male mating activity in the homozygous condition. The result suggests that more deleterious alleles on male mating activity may be hidden in the heterozygous condition in the third chromosome than in the second 
Table 1 Male mating activity of the four different genotypes at $F=0$ and $F=1$

\begin{tabular}{|c|c|c|c|c|c|}
\hline Genotype & $C y /+; U b x /+$ & $+\mid+; U b x /+$ & $C y /+;+/+$ & $+1+;+1+$ & Total \\
\hline \multicolumn{6}{|l|}{$F=0$} \\
\hline Females tested & 1291 & 1303 & 1096 & 1152 & 4842 \\
\hline Females mated & 970 & 1027 & 783 & 871 & 3651 \\
\hline Male mating activity & 0.751 & 0.788 & 0.714 & 0.756 & \\
\hline \multicolumn{6}{|l|}{$F=1$} \\
\hline Females tested & 2286 & 2359 & 2251 & 2327 & 9223 \\
\hline Females mated & 1738 & 1386 & 937 & 808 & 4869 \\
\hline Male mating activity & 0.760 & 0.588 & 0.416 & 0.347 & \\
\hline \multirow[t]{2}{*}{ Relative activity } & 1 & 0.764 & 0.647 & 0.552 & \\
\hline & & \pm 0.035 & \pm 0.052 & \pm 0.044 & \\
\hline
\end{tabular}

one. Second and third chromosomes examined for mating activity are not necessarily representative of the natural population because lethality and sterilityconferring chromosomes were omitted (see Materials and methods). From the mating activity in Table 1, the values of the two indices of epistatic interaction, $k$ and $i$, on the mean basis are estimated to be -0.027 and -0.097 , respectively.

Table 2 presents observed and expected male mating activities of the double chromosome homozygotes for 26 lines individually, with $k$ and $i$. The expected male mating activity is the product of mating activities of the single homozygotes under the multiplicative model. The mean value of the observed mating activity is $0.552 \pm 0.059$, whereas the expected activity is estimated to be $0.512 \pm 0.055$. These two values of male mating activities do not differ statistically, also indicating no, or almost no, epistasis between second and third chromosomes.

As shown in Table 2, the mean value of the $k$ statistic is calculated to be $0.001 \pm 0.0267$. The $k$ value is explicitly not different from zero. Negative $k$ statistics are obtained in just 13 lines out of 26 . The mean value of the coefficient of interaction $(i)$ is $0.1738 \pm 0.1678$. It is also not statistically different from zero owing to the large variance. As the range of $i$ statistic is $-1.0 \leqslant i \leqslant+1.0$, the mean value of $i$ is not so important. The median of the $i$ statistic is -0.012 and 0.020 . As $i$ is not symmetrically distributed about zero, the possibility that the chromosomal epistastic interaction is underestimated toward negative epistasis due to the disproportionate contribution of a few large negative $i$ values still remains. If the negative $i$ statistic $<-1.0$ is moderately fixed at $i=-1.0$, the mean $i$ value naturally increases to $-0.0367 \pm 0.0925$, which is also not significantly different from zero.

Taking these results together, it can be concluded that there is no interchromosomal epistatic interaction between two major autosomes for male mating activity of D. melanogaster under the condition of no competition among males for females.

Complete interchromosomal synthetic sterility was found in one line (Line No. 113) out of 26, where the $i$ statistic is 1.0 and the value of $k$ is 0.245 . Double chromosome homozygotes cannot mate, although both single homozygous males for second and third chromosomes have good fertility.

\section{Discussion}

The relative mating activities of each single homozygote for second and third chromosomes and the double homozygotes compared to $C y /+; \quad U b x /+$ heterozygotes in the previous study were $0.726 \pm 0.042, \quad 0.695 \pm 0.050$ and $0.517 \pm 0.064$, respectively (Kosuda, 1985). These activities are almost in agreement with those in the present study. As the difference in male mating activity between the two studies is not statistically different, it is interesting to deal with both results at once. Combined mating activities of each of the single homozygotes for second and third chromosomes and the double homozygotes gives $0.747 \pm 0.027, \quad 0.671 \pm 0.036$ and $0.526 \pm 0.044$, respectively. These combined activities are based on 49 chromosome lines and are estimated by the examination of more than 23,000 females in all. From these figures the means of the two indices of epistasis were quantified as $k=-0.012$ and $i=-0.060$. These indices also strongly favour the conclusion that there is almost no epistatic interaction between second and third chromosomes for male mating activity. Although chromosomes employed in the present and previous studies are not random samples from the natural population in Katsunuma, the deleterious effect of chromosomal homozygosis on male mating activity seems to be much greater in the second than in the third 
Table 2 Observed and expected mating activities of the double chromosome homozygotes and two indices of epistasis, $i$ and $k$

\begin{tabular}{|c|c|c|c|c|}
\hline Line & $\begin{array}{l}\text { Observed } \\
\text { activity }\end{array}$ & $\begin{array}{l}\text { Expected } \\
\text { activity }\end{array}$ & $k$ & $i$ \\
\hline 101 & 0.731 & 0.883 & 0.047 & 0.172 \\
\hline 102 & 0.887 & 0.829 & 0.017 & -0.070 \\
\hline 103 & 0.772 & 0.733 & -0.013 & -0.053 \\
\hline 105 & 0.596 & 0.159 & -0.299 & $-2.748^{*}$ \\
\hline 106 & 0.625 & 0.370 & -0.127 & -0.689 \\
\hline 107 & 0.538 & 0.503 & -0.016 & -0.070 \\
\hline 108 & 0.796 & 0.553 & -0.079 & -0.374 \\
\hline 109 & 0.600 & 0.572 & -0.011 & -0.049 \\
\hline 110 & 0.386 & 0.394 & 0.009 & 0.020 \\
\hline 111 & 0.095 & 0.389 & 0.250 & 0.755 \\
\hline 112 & 0.678 & 0.204 & 0.028 & 0.127 \\
\hline 113 & 0 & 0.217 & 0.311 & 1.000 \\
\hline 114 & 0.710 & 0.186 & -0.309 & $-2.817^{*}$ \\
\hline 116 & 0.104 & 0.405 & 0.248 & 0.743 \\
\hline 118 & 1.008 & 1.038 & 0.007 & 0.029 \\
\hline 124 & 0.397 & 0.575 & 0.088 & 0.310 \\
\hline 131 & 0.512 & 0.590 & 0.034 & 0.132 \\
\hline 132 & 1.193 & 1.060 & -0.029 & -0.125 \\
\hline 133 & 0.544 & 0.656 & 0.046 & 0.171 \\
\hline 134 & 0.372 & 0.232 & -0.106 & -0.603 \\
\hline 135 & 0.349 & 0.377 & 0.018 & 0.074 \\
\hline 136 & 0.696 & 0.618 & -0.030 & -0.126 \\
\hline 137 & 0.086 & 0.093 & 0.012 & 0.073 \\
\hline 138 & 0.503 & 0.564 & 0.028 & 0.108 \\
\hline 139 & 0.250 & 0.167 & -0.084 & -0.497 \\
\hline 140 & 0.963 & 0.952 & -0.003 & -0.012 \\
\hline \multirow[t]{2}{*}{ Mean } & $\begin{array}{r}0.552 \\
\pm 0.059\end{array}$ & $\begin{array}{r}0.512 \\
\pm 0.055\end{array}$ & $\begin{array}{r}0.0001 \\
+0.0267\end{array}$ & $\begin{array}{r}-0.1738 \\
+0.1678\end{array}$ \\
\hline & & & & \\
\hline
\end{tabular}

*When these two values of $i<-1.0$ are moderately fixed at $i=-1.0$, mean $i=-0.003 \pm 0.093$.

chromosome. Kosuda (1971) also reported a more deleterious effect of the third chromosome than the second on egg-to-adult viability of $D$. melanogaster.

Two indices of interchromosomal epistatic interaction, $k$ and $i$, in the previous study were $0.029 \pm 0.027$ and $0.008 \pm 0.129$, respectively. Since the $k$ statistic is in agreement with the present one, they have been combined and are presented in Table 3 . The amalgamated $k$ and $i$ statistics of present and previous studies were disclosed to be $0.014 \pm 0.019$ and $-0.088 \pm 0.108$, respectively. Both $k$ and $i$ statistics do not statistically differ from $0 . k$ has a mean negative value whereas $i$ is positive, although $k$ and $i$ indices of epistasis should essentially have the same sign. The fact also indicates that there is almost no epistatic interaction for male mating activity. 27 chromosome lines out of 49 have positive $k$ and $i$ values, with negative epistasis observed in the remaining 22 lines, not statistically different from a 1:1 segregation. Observed and expected mating activities in all are $0.526 \pm 0.044$ and $0.511 \pm 0.038$, respectively. The coincidence between these two combined activities is excellent. These results imply that there is almost no epistatic interaction between second and third chromosomes for male mating activity.

Note that the sample of chromosome pairs is not a random sample of the natural population because chromosomes with poor viability were excluded from the experiment. Chromosomes with alleles for low mating activity and sterility alleles were also omitted for the following reasons. It is very difficult to estimate an accurate mating activity due to the large variance for chromosomes carrying alleles for low mating activity. Unless an extreme negative epistasis exists, chromosome pairs with sterility alleles give no interaction $(i=0)$, as Crow (1968) pointed out. Consequently, the frequency of sterility alleles in both chromosomes is higher, and the mean $i$ is more biased towards zero. Thus the magnitude of epistatic interaction is influenced by the numbers of sterility alleles in the population, if sterility chromosomes are included. As the results of the present study refer to a subset of chromosomes in the population, the question whether the apparent absence of interchromosomal epistatic interaction is also true of the excluded chromosome still remains unsolved. It is probable that considerable epistasis exists between chromosomes that cause a drastic reduction in male mating activity. In fact, Kosuda \& Moriwaki $(1968,1971)$ reported that some lethals may be the result of the synergistic interactions of severely deleterious alleles influencing egg-to-adult viability of D. melanogaster.

Interchromosomal synthetic sterility was found in one chromosome line out of 26 in the present experiment. Kosuda (1985) also reported complete synthetic sterility between chromosomes in one out of 23 chromosome lines. In this chromosome line (Line No. $009), i$ is 1.0 and $k$ amounts to as much as 0.311 . The frequency of interchromosomal synthetic sterility is $0.04(=2 / 49)$ in total. Synthetic lethality between second and third chromosomes of $D$. melanogaster was also reported by Kosuda (1971). Although single chromosome homozygotes for second and and third chromosomes have good viabilities, two double chromosome homozygotes out of 267 were lethal. The frequency of interchromosomal synthetic sterility seems to be much higher than that of interchromosomal synthetic lethality. However, the generality of synthetic lethals within chromosomes is strongly controversial, as it is difficult to verify its existence (Kosuda \& Moriwaki, 1968, 1971; Thompson, 1986). 
Table 3 Summarized results for observed and expected mating activity of the double chromosome homozygotes and two indices of epistasis, $k$ and $i$, in the present and previous studies

\begin{tabular}{lcrr}
\hline & Present study & Previous study & \multicolumn{1}{c}{ Combined } \\
\hline Observed activity & $0.552 \pm 0.059$ & $0.517 \pm 0.064$ & $0.526 \pm 0.044$ \\
Expected activity & $0.512 \pm 0.055$ & $0.509 \pm 0.054$ & $0.511 \pm 0.038$ \\
$k$ statistic & $0.0001 \pm 0.0267$ & $0.029 \pm 0.027$ & $0.014 \pm 0.019$ \\
$i$ statistic & $-0.174 \pm 0.168$ & $0.008 \pm 0.129$ & $-0.088 \pm 0.108$ \\
$i$ statistic* & $-0.037 \pm 0.093$ & $-0.036 \pm 0.116$ & $-0.003 \pm 0.073$ \\
\hline
\end{tabular}

$*_{i}$ statistic when $i$ is less than -1.0 is moderately fixed $i=-1.0$.

The egg-to-adult viability of Drosophila flies simultaneously homozygous for second and third chromosomes was compared with single homozygotes for second or third chromosomes to determine whether different gene loci have independent effects (Spassky et al., 1965; Temin et al., 1969; Kosuda, 1971). The conclusion from these studies is that double homozygote viability is, in general, slightly lower than that expected from two single homozygotes under the multiplicative model; that is, there is a weak synergistic or positive interchromosomal interaction. These studies examined exclusively egg-to-adult viability as a measure of fitness, and fitness components other than preadult viability have been neglected in this kind of approach. However, a body of evidence has accumulated to support the fact that mating activity has a much greater effect on total fitness than egg-to-adult viability (Anderson, 1969; Petit et al., 1980; Brittnacher, 1981; Sharp, 1982; Kosuda, 1983).

Seager et al. (1982) reported a strong diminishing or negative epistatic interaction $(k=0.352 \pm 0.210)$ for total fitness in an artificial cage population of Drosophila melanogaster. If the synergistic or no epistatic interaction exists for egg-to-adult viability, as has repeatedly been reported, a conspicuously strong diminishing epistasis is expected in male mating activity. However, no evidence for negative epistasis for male mating activity could be detected in this or previous studies. Reasons for the discrepancy have not been found yet but may result from the differences in experimental conditions and methods. Extremely severe competition, especially in the larval stages, is suspected in cage populations and this may accelerate negative epistatic interaction.

Clark \& Feldman (1981) investigated interchromosomal interaction in the heterozygous condition for various fitness components between two second chromosomes with specific inversions marked by $C y$ and $P m$, respectively. They could not detect consistent epistatic interaction for male mating success. Inter- action was significantly positive in one case and was significantly negative in another, whereas it was not significant in a further four cases.

Different types of study to clarify intrachromosomal epistatic interaction for various fitness components have been carried out in several species of Drosophila and other insects (Dobzhansky et al., 1963; Malogolowkin-Cohen et al., 1964; Mukai, 1969; Kosuda, 1972, 1980). If different gene loci have independent fitness effects, then the relationship between the degree of inbreeding and the fitness depression should be linear on the logarithmic scale. Latter \& Robertson (1962) showed no significant deviation from linearity between the rate of inbreeding and the reduction in the competitive male mating activity of Drosophila melanogaster across a wide range of inbreeding coefficients.

Furthermore, Sharp (1984) also found the linear relationship under the additive model. In this kind of approach to the problem, however, successive matings are inevitable. Consequently, the coefficient of inbreeding would be overestimated, especially in the later stages of successive matings, and epistatic interaction would be underestimated in a positive direction. These reports supporting a lack of intrachromosomal epistasis are not inconsistent with the conclusion of the present study.

\section{Acknowledgements}

The author wishes to express his thanks to one anonymous reviewer for making useful suggestions and also to the President of Josai University for financial support.

\section{References}

ANDERSON, w. w. 1969. Selection in experimental populations. I. Lethal genes. Genetics, 62, 653-672. 
BRITTNACHER, J. C. 1981. Genetic variation and genetic load due to the male reproductive component of fitness in Drosophila. Genetics, 97, 719-730.

CLARK, A. G. AND FELDMAN, M. W. 1981. The estimation of epistasis in components of fitness in experimental populations of Drosophila melanogaster. II. Assessment of meiotic drive, viability, fecundity and sexual selection. Heredity, 46, 347-377.

CROW, J. F. 1968. Some analyses of hidden variability in Drosophila populations. In: Lewontin, R. C. (ed.) Population Biology and Evolution, Syracuse Univ. Press, pp. 71-86.

DOBZHANSKY, TH., SPASSKY, B. AND TIDWELL, T. 1963. Genetics of natural populations. XXXII. Inbreeding and the mutational and balanced genetic loads in natural populations of Drosophila pseudoobscura. Genetics, 48, 361-373.

KOSUDA, K. 1971. Synergistic interaction between second and third chromosomes on viability of Drosophila melanogaster. Jpn J. Genet., 46, 41-52.

KosUDA, K. 1972. Synergistic effect of inbreeding on viability in Drosophila virilis. Genetics, 72, 461-468.

KoSUDA, K. 1980. The effect of inbreeding on viability in Drosophila simulans. Jpn J. Genet., 55, 307-310.

KOSUDA, K. 1983. Genetic variability in mating activity of Drosophila melanogaster males. Experientia, 39, 100-101.

KOSUDA, K. 1985. Effects of chromosomal interaction on male mating activity in Drosophila melanogaster. Jpn J. Genet., 60, 63-70.

KOSUDA, K. AND MORIWAKI, D. 1968. 'Synthetic lethals' in the second chromosomes of Drosophila melanogaster. Proc Jpn Acad., 44, 833-836.

KOSUDA, K. AND MORIWAKI, D. 1971. Increase of genetic variability through recombination in Drosophila melanogaster. Genetics, 67, 287-304.
LATTER, B. D. H. AND ROBERTSON, A. 1962. The effect of inbreeding and artificial selection on reproductive fitness. Genet. Res. Camb., 3, 110-138.

MALOGOLOWKIN-COHEN, CH., LEVENE, H., DOBZHANSKY, N. P. AND SIMMONS, A. S. 1964. Inbreeding and the mutational and balanced loads in natural populations of Drosophila pseudoobscura. Genetics, 50, 1299-1311.

MUKAI, T. 1969. The genetic structure of natural populations of Drosophila melanogaster. VII. Synergistic interaction of spontaneous mutant polygenes controlling viability. Genetics, 61, 749-761.

PETIT, C. P., BOURGEERON, P. AND MERCOT, H. 1980. Multiple mating, effective population size, and sexual selection in Drosophila melanogaster. Heredity, 45, 281-292.

PROUT, T. 1965. The estimation of fitness from genotypic frequencies. Evolution, 19, 546-561.

SEAGER, R. D. AND AYALA, F. 1982. Chromosome interaction in Drosophila melanogaster. I. Viability studies. Genetics, 102, 467-483.

SEAGER, R. D., AYAlA, F. AND MARKs, R. W. 1982. Chromosome interaction in Drosophila melanogaster. II. Total fitness. Genetics, 102, 485-502.

SHARP, P. M. 1982. Competitive mating in Drosophila melanogaster. Genet. Res. Camb., 40, 201-205.

SHARP, P. M. 1984. The effect of inbreeding on competitive male-mating ability in Drosophila melanogaster. Genetics, 106, 601-612.

SPASSKY, B., DOBZHANSKY, TH. AND ANDERSON, w. w. 1965. Genetics of natural populations. XXXVI. Epistatic interactions of the components of the genetic load in Drosophila pseudoobscura. Genetics, 52, 653-664.

TEMIN, R. G., MEYER, H. U. DOWSON, P. S. AND CROW, J. F. 1969. The influence of epistasis on homozygous viability depression in Drosophila melanogaster. Genetics, 61, 497-519.

THOMPSON, v. 1986. Half-chromosome viability and synthetic lethality in Drosophila melanogaster. J. Hered., 77, 385-388. 